\title{
The results on vertex domination in Fuzzy graphs
}

\author{
Mohammadesmail Nikfar \\ Department of Mathematics, Payame Noor University, P. O. Box: 19395-3697, Tehran, \\ Iran \\ *m.s.nikfar@member.ams.org; m.s.nikfar@gmail.com
}

\begin{abstract}
We do fuzzification the concept of domination in crisp graph by using membership values of nodes, $\alpha$-strong and arcs. In this paper, we introduce a new variation on the domination theme which we call vertex domination. We determine the vertex domination number $\gamma_{v}$ for several classes of fuzzy graphs, specially complete fuzzy graph and complete bipartite fuzzy graphs. The bounds is obtained for the vertex domination number of fuzzy graphs. Also the relationship between $M$-strong arcs and $\alpha$-strong is obtained. In fuzzy graphs, monotone decreasing property and monotone increasing property is introduced. We prove the vizing's conjecture is monotone decreasing fuzzy graph property for vertex domination. we prove also the Grarier-Khelladi's conjecture is monotone decreasing fuzzy graph property for it. We obtain Nordhaus-Gaddum (NG) type results for these parameters. The relationship between several classes of operations on fuzzy graphs with the vertex domination number of them is studied.
\end{abstract}

Keywords: Fuzzy graph, $\alpha$-strong arcs, Weight of nodes, vertex domination AMS Subject Classification: 05C72, 05C69, 03E72, 94D05

\section{Introduction}

L.A. Zadeh introduced the concept of a fuzzy subset of a set as a way for representing uncertainty. Zadeh's ideas stirred the interest of researchers worldwide. His ideas have been applied to a wide range of scientific areas. Theoretical mathematics has also been touched by the notion of a fuzzy subset. In 1965, Zadeh published his seminal paper "fuzzy sets" [77] which described fuzzy set theory and consequently fuzzy logic. The purpose of Zadeh's paper was to develop a theory which could deal with ambiguity and imprecision of certain classes or sets in Human thinking, particularly in the domains of pattern recognition, communication of information, and observation. This theory proposed making the grade of membership of an element in a subset of a universal set a value in the closed interval $[0,1]$ of real numbers. Zadeh's idea have found applications in computer science, artificial intelligence, decision analysis, information science, system science, control engineering, expert systems, pattern recognition, management science, operations research, and robotics. Theoretical mathematics has also been touched by fuzzy set theory. In the classical set theory introduced by Cantor, values of elements in a set are either 0 or 1 . That is for any element, there are only two possibilities: the element is the set or it is not. Therefore, Cantor set theory cannot handle data with ambiguity and uncertainty. The ideas of fuzzy set theory have been introduced into topology, abstract algebra, geometry, graph theory, and analysis. Analytical 
representation of physical phenomena can be fruitful as models of reality, but are sometimes difficult to understand because they do not explain much by themselves, and may remain unclear to the non-specialist. In other words, Zadeh proposed fuzzy theory and introduced fuzzy set theory which can be considered as the phenomenon of ambiguity across all systems displaying this property and its consequences.

Graph theory is one of the branches of modern mathematics having experienced a most impressive development in recent years. The origin of graph theory can be traced back to Euler's work on the Konigsberg bridge problem (1735) which subsequently led to the concept of an Eulerian graph. The first text book on graph theory was written by D'enesKonig and published in 1936. A later text book by Frank Harary published in 1968, was enormously popular and enabled mathematicians, chemists, electrical engineers and social scientists to have common platform to dialogue with each other. Graphs are represented graphically by taking a set of points on the plane and it is desired to find some structure among the points in the form of edges containing a subset of the pair of points. Graph theory plays a vital role as far as application side is concerned. Graph theory is intimately related to many branches of mathematics including group theory, matrix theory, numerical analysis, probability, topology and combinatorics because of its diagrammatic representation and its intuitive and aesthetic appeal.

One of the most interesting problems in graph theory is that of Domination Theory. The earliest ideas of dominating sets are found in the classical problems of covering chess board with minimum number of chess pieces. Nowadays domination theory ranks top among the most prominent areas of research in graph theory and combinatorics. The concept of domination in graphs, with its many variations, is now well studied in graph theory. The book by Chartrand and Lesniak [16] includes a chapter on domination. For a more thorough study of domination in graphs, see Haynes et al. [24]. The current list of papers on domination in [24] has over 1200 entries. The theory of domination is formalized by Clauge Berge in his book "Theory of graphs and its application" (1962). Berge mentions the strategies of keeping a number of locations under surveillance, by a set of radar station. Oystein Ore was a first person to use the term domination number in his book on Graph Theory. The theory of domination has been the nucleus of research activity in graph theory in recent times. The fastest growing area within graph theory is a study of domination and related subset problems such independence, covering, matching, decomposition and labelling. Domination boasts a host of applications to social network theory, land surveying, game theory, interconnection network, parallel computing and image processing and so on. Today, this theory gained popularity and remains as a major area of research due to the contributions of O.Ore [50], C.Berge [6], E.J.Cockayne [19], S.T.Hedetniemi [24], T.W.Haynes [24], R.C.Laskar [25], P.J.Slater [66], V.R.Kulli [32], E.Sampathkumar [62], S.Arumugam [4].

Fuzzy graph theory has numerous applications in various fields like clustering analysis, database theory, network analysis, information theory, etc. [44]. Fuzzy models can be used in problems handling uncertainty to get more accurate and precise solutions [74-76]. As in graphs, connectivity concepts play a key role in applications related with fuzzy graphs $[44,70]$. The fuzzy definition of fuzzy graphs was proposed by Kaufmann [28], from the fuzzy relations introduced by Zadeh. Although Rosenfeld introduced another elaborated definition, including fuzzy vertex and fuzzy edges. Fuzzy graphs were introduced by Rosenfeld [56] and Yeh and Bang [73] independently in 1975. Rosenfeld in his paper "Fuzzy Graphs" presented the basic structural and connectivity concepts while Yeh and Bang introduced different connectivity parameters of a fuzzy graph and discussed their applications in the paper titled "Fuzzy relations, Fuzzy graphs and their applications to clustering analysis" [73]. Rosenfeld considered fuzzy relations on fuzzy sets and developed the structure of fuzzy graphs, obtaining analogues 
of several graph theoretical concepts. He introduced and examined such concepts as paths, connectedness and clusters, bridges, cut vertices, forests and trees. Fuzzy graphs introduced by Rosenfeld are finding an increasing number of applications in modelling real time systems where the level of information inherent in the system varies with different levels of precision. Fuzzy models are becoming useful because of their aim in reducing the difference between the traditional numerical models used in engineering and sciences and the symbolic models used in expert systems and AI. After the pioneering work of Rosenfeld and Yeh and Bang in 1975, when some basic fuzzy graph theoretic concepts and applications have been indicated, several authors have been finding deeper results, and fuzzy analogues of many other graph theoretic concepts. This include fuzzy trees [21,67], fuzzy line graphs [42], operations on fuzzy graphs [43], automorphism of fuzzy graphs [9,11], fuzzy interval graphs [20], cycles and cocycles of fuzzy graphs [45], and metric aspects in fuzzy graphs [65]. Bhutani and Rosenfeld have introduced the concept of strong arcs [12]. Different parameters like sum distance in fuzzy graphs and chromatic number of fuzzy graphs were discussed in [29,69]. The work on fuzzy graphs was also done by Akram, Samanta, Nayeem, Pramanik, Rashmanlou and Pal [1-3,48,51-55,57-61]. P.Bhattacharya [10] discussed some properties of fuzzy graphs and introduced the notion of eccentricity and centre in fuzzy graphs.

K.R.Bhutani [13] introduced the concept of complete fuzzy graphs and concluded that a complete fuzzy graph has no cut nodes. $\mathrm{Xu}$ [72] applied connectivity parameters of fuzzy graphs to problems in chemical structures.

The concept of domination in fuzzy graphs was investigated by A.Somasundaram and S.Somasundaram. A.Somasundaram presented the concepts of independent domination, total domination, connected domination and domination in cartesian products and composition of fuzzy graphs [63]. Somasundaram and Somasundaram discussed domination in fuzzy graphs. They defined domination using effective edges in fuzzy graph $[63,64]$. Nagoorgani and Chandrasekharan defined domination in fuzzy graphs using strong arcs [47]. Manjusha and Sunitha discussed some concepts in domination and total domination in fuzzy graphs using strong arcs [36,37]. A. Selvam Avadayappan, G. Mahadevan, A. Mydeenbibi, T.A. Subramanian, A. Nagarajan, A. Rajeswari have studied the problem of obtaining an upper bound for the sum of a domination parameter and a graph theoretic parameter and characterized the corresponding extremal graphs.

Motivated by the notion of dominating sets and their applicability, we focused on introducing some dominating parameters in fuzzy graph theory. For fuzzification of the following problems, types of nodes (based on advantages) and types of connection with nodes can be assigned by different values. So the question is based on based on values on nodes and ratio of total of values of adjacent $\alpha$-strong connections to total of values of adjacent connections?

Chess enthusiasts in Europe considered the problem of determining the minimum number of queens that can be placed on a chess board so that all the squares are either attacked by a queen or occupied by a queen. Harary et al. [5] explained an interesting application in voting situations using the concept of domination. A number of strategic locations are to be kept under observations. One of the important areas of applications of domination is communication network, where a dominating set represents a set of cities which, acting as transmitting stations, can transmit messages to every city in the network. Another area of application of domination is voting situations. Suppose the commander of the Army Postal services plans to set up a few post offices in an important region with minimum number of post offices to control the whole region. Now-a-day almost all schools operate school buses for transporting children to and from schools. Among many points, three important points to be noted are 1. The running time of a bus between school and its terminus. 2. Maximum number of students in a 
bus at any one time and 3. The maximum distance a student has to walk to board a school bus. Consider a computer network modeled by a 4 -cube. The vertices of the 4-cube represents computers and edges represent direct communication link between two computers. So, in this model we have 16 computers or processers to which it is directly connected. The problem is to collect information from all processors and we like to do it relatively often and relatively fast. So we identify a small set of processors called collecting processors and ask each processor to send its information to one of a small set of collecting processors. We assume that at most a one-unit delay between the time a processor sends its information and time it arrives at a nearest collector is allowed. So, we have to find an dominating set among the set of a processors. Consider the problem of locating a single fire station, police station or a similar such service facility to serve the communities. Also, we would like to locate such a service facility in one of these communities and not at an arbitrary point along the road, due to some reasons. Let $P_{n}$ be a set of points in general position on the plane. The unit distance graph $U D G\left(P_{n}\right)$ associated to $P_{n}$ is a graph whose vertex set consists of the elements of $P_{n}$, two of which are connected if they are at distance at most one. Unit distance graphs are used to model various types of wireless networks, including cellular networks, sensor networks, ad-hoc networks and others in which the nodes represent broadcast stations with a uniform broadcast range we shall refer to networks that can be modeled using unit distance graphs as unit distance wireless networks, abbreviated as UDW networks.

We first briefly illustrate our opinion. The rest of this paper is organized as follows. In Section 2, we lay down the preliminary results which recall some basic concepts of fuzzy graph, path, cycle, connectedness, complete fuzzy graph, order, size, complement, types of arcs consists of $\alpha$-strong, $\beta$-strong, $\delta$-strong and $M$-strong, bipartite fuzzy graph, complete bipartite fuzzy graph, star fuzzy graph, be isolated, domatic partition, Vizing's conjecture, Gravier and Khelladi's conjecture, some operations on fuzzy graphs consists of cartesian product, join and union, Nordhaus-Gaddum (NG) results and finally we conclude this section with Remark (2.1) and In Section 3, The $\alpha$-strong domination number of a fuzzy graph is defined in a classic way, Definition (3.1), (3.3), (3.4). We determine vertex domination number for several classes of fuzzy graphs consists of complete fuzzy graph, Proposition (3.10), empty fuzzy graph, Proposition (3.11), star fuzzy graph, Proposition (3.13), complete bipartite fuzzy graph, Proposition (3.14). We give an upper bound for the vertex domination number of fuzzy graphs, Proposition (3.15). For any fuzzy graph the Nordhaus-Gaddum(NG)'result holds, Theorem (3.16). Finding domatic partition of size two in fuzzy graph $G$ of order $n \geq 2$ is studied, Theorem (3.19). We improve upper bound for the vertex domination number of fuzzy graphs without isolated nodes, Theorem (3.20). We also improve

Nordhaus-Gaddum(NG)'result for fuzzy graphs without isolated nodes, Corollary (3.21). We give the relationship between $M$-strong arcs and $\alpha$-strong arcs, Corollary (3.24). We give a necessary and sufficient condition for vertex domination which is half of order, In fact fuzzy graphs with vertex domination which is half of order is characterized in the special case, Theorem (3.26). The vertex domination of union of two fuzzy graphs is studied, Proposition (3.27). Also the vertex domination of union of fuzzy graphs Family is discussed, Corollary (3.28). The concepts of both monotone increasing fuzzy graph property, Definition (3.29), and monotone decreasing fuzzy graph property, Definition (3.31), are introduced. The result in relation with vizing's conjecture by using $\alpha$-strong arc and monotone decreasing fuzzy graph property is determined, Theorem (3.34). Some results in relation with vizing's conjecture by using $\alpha$-strong arc and spanning fuzzy subgraph is studied, Corollary (3.35). The vertex domination of join of two fuzzy graphs is studied, Proposition (3.36). Also the vertex domination of join of fuzzy graphs Family is discussed, Corollary (3.37). The result in relation with Gravier and Khelladi's conjecture by using $\alpha$-strong arc and monotone decreasing fuzzy graph property is 
determined, Theorem (3.38). We conclude this section with Some result in relation with Gravier and Khelladi's conjecture by using $\alpha$-strong arc and spanning fuzzy subgraph is studied, Corollary (3.39). In Section 4, We give 9 practical applications in relation with these concepts.

\section{Preliminary}

We provide some basic background for the paper in this section.

Some of the books discussing these various themes are Bezdek and Pal [7], Lootsma [35], Morderson and Malik [40], Comelius . T. Leondes [34] and Klir and Bo Yuan [31]. We shall now list below some basic definitions and results from [41], [56]. Also Background on fuzzy graphs and the following definitions can be found in them.

we lay down the preliminary results which recall some basic concepts of fuzzy graph, path, cycle, connectedness, complete fuzzy graph, order, size, complement, types of arcs consists of $\alpha$-strong, $\beta$-strong, $\delta$-strong and $M$-strong, bipartite fuzzy graph, complete bipartite fuzzy graph, star fuzzy graph, be isolated, domatic partition, Vizing's conjecture, Gravier and Khelladi's conjecture, some operations on fuzzy graphs consists of cartesian product, join and union, Nordhaus-Gaddum (NG) results and finally we conclude this section with Remark (2.1)

We recall that a fuzzy subset of a set $\mathrm{S}$ is a function of $\mathrm{S}$ into the closed interval $[0$, 1], [77]. A fuzzy graph is denoted by $G=(V, \sigma, \mu)$ such that $\mu(\{x, y\}) \leq \sigma(x) \wedge \sigma(y)$ for all $x, y \in V$ where $V$ is a vertex set, $\sigma$ is a fuzzy subset of $V$ and $\mu$ is a fuzzy relation on $V$. We call $\sigma$ the fuzzy node set (or fuzzy vertex set) of $G$ and $\mu$ the fuzzy arc set (or fuzzy edge set) of $G$, respectively. We consider fuzzy graph $G$ with no loops and assume that $V$ is finite and nonempty, $\mu$ is reflexive (i.e., $\mu(\{x, x\})=\sigma(x)$, for all $x$ ) and symmetric (i.e., $\mu(\{x, y\})=\mu(\{y, x\})$, for all $x, y \in V)$. In all the examples $\sigma$ and $\mu$ is chosen suitably. In any fuzzy graph, the underlying crisp graph is denoted by $G^{*}=(V, E)$ where $V$ and $E$ are domain of $\sigma$ and $\mu$, respectively. This definition of fuzzy graph is essentially the same as the one appearing in [56]. The fuzzy graph $H=(\tau, \nu)$ is called a partial fuzzy subgraph of $G=(\sigma, \mu)$ if $\nu \subseteq \mu$ and $\tau \subseteq \sigma$. Similarly, the fuzzy graph $H=(\tau, \nu)$ is called a fuzzy subgraph of $G=(V, \sigma, \mu)$ induced by $P$ if $P \subseteq V, \tau(x)=\sigma(x)$ for all $x \in P$ and $\nu(\{x, y\})=\mu(\{x, y\})$ for all $x, y \in P$. For the sake of simplicity, we sometimes call $H$ a fuzzy subgraph of $G$. We say that the partial fuzzy subgraph $(\tau, \nu)$ spans the fuzzy graph $(\sigma, \mu)$ if $\sigma=\tau$. In this case, we call $(\tau, \nu)$ a spanning fuzzy subgraph of $(\sigma, \mu)$.

For the sake of simplicity, we sometimes write $x y$ instead of $\{x, y\}$

A path $P$ of length $n$ is a sequence of distinct nodes $u_{0}, u_{1}, \cdots, u_{n}$ such that $\mu\left(u_{i-1}, u_{i}\right)>0, i=1,2, \cdots, n$ and the degree of membership of a weakest arc is defined as its strength. If $u_{0}=u_{n}$ and $n \geq 3$ then $P$ is called a cycle and $P$ is called a fuzzy cycle, if it contains more than one weakest arc. The strength of a cycle is the strength of the weakest arc in it. The strength of connectedness between two nodes $x$ and $y$ is defined as the maximum of the strengths of all paths between $x$ and $y$ and is denoted by $C O N N_{G}(x, y)$.

A fuzzy graph $G=(V, \sigma, \mu)$ is connected if for every $x, y$ in $V, C O N N_{G}(x, y)>0$.

An arc $u v$ of a fuzzy graph is called an $M$-strong arc if $\mu(u v)=\sigma(u) \wedge \sigma(v)$. In order to avoid confusion with the notion of strong arcs introduced by Bhutani and Rosenfeld [15], we shall call strong in the sense of Mordeson as M-strong [46].

A fuzzy graph $G$ is said complete if $\mu(u v)=\sigma(x) \wedge \sigma(y)$. for all $u, v \in V$.

The order $p$ and size $q$ of a fuzzy graph $G=(V, \sigma, \mu)$ are defined $p=\Sigma_{x \in V} \sigma(x)$ and $q=\Sigma_{x, y \in V} \mu(x y)$.

The complement of a fuzzy graph $G$, denoted by $\bar{G}$ is defined to $\bar{G}=(V, \sigma, \bar{\mu})$ where $\bar{\mu}(x y)=\sigma(x) \wedge \sigma(y)-\mu(x y)$ for all $x, y \in V$. 
An arc of a fuzzy graph is called $\alpha$-strong if its weights is greater than strength of connectedness of its end nodes when it is deleted. Depending on $C O N N_{G}(x, y)$ of an arc $x y$ in a fuzzy graph $G$, Mathew and Sunitha [68] defined three types of arcs. Note that $C O N N_{G-x y}(x, y)$ is the strength of connectedness between $x$ and $y$ in the fuzzy graph obtained from $G$ by deleting the arc $x y$. An arc $x y$ in $G$ is $\alpha$-strong if $\mu(x y)>C O N N_{G-x y}(x, y)$. An arc $x y$ in $G$ is $\beta$-strong if $\mu(x y)=C O N N_{G-x y}(x, y)$. An arc $x y$ in $G$ is $\delta$-arc if $\mu(x y)<C O N N_{G-x y}(x, y)$.

A fuzzy graph $G$ is said bipartite if the vertex set $V$ can be partitioned into two nonempty sets $V_{1}$ and $V_{2}$ such that $\mu\left(v_{1} v_{2}\right)=0$ if $v_{1}, v_{2} \in V_{1}$ or $v_{1}, v_{2} \in V_{2}$. Moreover, if $\mu(u v)=\sigma(u) \wedge \sigma(v)$ for all $u \in V_{1}$ and $v \in V_{2}$ then $G$ is called a complete bipartite graph and is denoted by $K \sigma_{1}, \sigma_{2}$, where $\sigma_{1}$ and $\sigma_{2}$ are respectively the restrictions of $\sigma$ to $V_{1}$ and $V_{2}$. In this case, If $\left|V_{1}\right|=1$ or $\left|V_{2}\right|=1$ then the complete bipartite graph is said a star fuzzy graph which is denoted by $K_{1, \sigma}$.

A node $u$ is said isolated if $\mu(u v)=0$ for all $v \neq u$.

A domatic partition is a partition of the vertices of a graph into disjoint dominating sets. The maximum number of disjoint dominating sets in a domatic partition of a graph is called its domatic number.

In graph theory, Vizing's conjecture [17] concerns a relation between the domination number and the cartesian product of graphs. This conjecture was first stated by Vadim G. Vizing (1968), and states that, if $\gamma(G)$ denotes the minimum number of vertices in a dominating set for $\mathrm{G}$, then

$$
\gamma(G \square H) \geq \gamma(G) \gamma(H)
$$

Vizing's conjecture from 1968 asserts that the domination number of the. Cartesian product of two graphs is at least as large as the product of their domination numbers.

Gravier and Khelladi (1995) conjectured a similar bound for the domination number of the tensor product of graphs; however, a counterexample was found by Klav ̌ar Zmazek (1996) [30]. Since Vizing proposed his conjecture, many mathematicians have worked on it, with partial results described below. For a more detailed overview of these results, see Brešar et al. (2012) [8]

The cartesian product $G=G_{1} \times G_{2}$ [39] of two fuzzy graphs $G_{i}=\left(V_{i}, \sigma_{i}, \mu_{i}\right), i=1,2$ is defined as a fuzzy graph $G=\left(V \times V, \sigma_{1} \times \sigma_{2}, \mu_{1} \times \mu_{2}\right)$ where $E=\left\{\left\{u u_{2}, u v_{2}\right\} \mid u \in V_{1}, u_{2} v_{2} \in E_{2}\right\} \cup\left\{\left\{u_{1} w, v_{1} w\right\} \mid u_{1} v_{1} \in E_{1}, w \in V_{2}\right\}$. Fuzzy sets $\sigma_{1} \times \sigma_{2}$ and $\mu_{1} \times \mu_{2}$ are defined as $\left(\sigma_{1} \times \sigma_{2}\right)\left(u_{1}, u_{2}\right)=\sigma_{1}\left(u_{1}\right) \wedge \sigma_{2}\left(u_{2}\right)$ and $\forall u \in V_{1}, \forall u_{2} v_{2} \in E_{2},\left(\mu_{1} \times \mu_{2}\right)\left(\left\{u u_{2}, u v_{2}\right\}\right)=\sigma_{1}(u) \wedge \mu_{2}\left(u_{2} v_{2}\right)$ and $\forall u_{1} v_{1} \in E_{1}, \forall w \in V_{2},\left(\mu_{1} \times \mu_{2}\right)\left(\left\{u_{1} w, v_{w}\right\}\right)=\mu_{1}\left(u_{1} v_{1}\right) \wedge \sigma_{2}(w)$.

The union $G=G_{1} \cup G_{2}$ [39] of two fuzzy graphs $G_{i}=\left(V_{i}, \sigma_{i}, \mu_{i}\right), i=1,2$ is defined as a fuzzy graph $G=\left(V_{1} \cup V_{2}, \sigma_{1} \cup \sigma_{2}, \mu_{1} \cup \mu_{2}\right)$ where $E=E_{1} \cup E_{2}$. Fuzzy sets $\sigma_{1} \cup \sigma_{2}$ and $\mu_{1} \cup \mu_{2}$ are defined as $\left(\sigma_{1} \cup \sigma_{2}\right)(u)=\sigma_{1}(u)$ if $u \in V_{1}-V_{2},\left(\sigma_{1} \cup \sigma_{2}\right)(u)=\sigma_{2}(u)$ if $u \in V_{2}-V_{1}$, and $\left(\sigma_{1} \cup \sigma_{2}\right)(u)=\sigma_{1}(u) \vee \sigma_{2}(u)$ if $u \in V_{1} \cap V_{2}$. Also $\left(\mu_{1} \cup \mu_{2}\right)(u v)=\mu_{1}(u v)$ if $u v \in E_{1}-E_{2}$ and $\left(\mu_{1} \cup \mu_{2}\right)(u v)=\mu_{2}(u v)$ if $u v \in E_{2}-E_{1}$, and $\left(\mu_{1} \cup \mu_{2}\right)(u v)=\mu_{1}(u v) \vee \mu_{2}(u v)$ if $u v \in E_{1} \cap E_{2}$.

Let $G=G_{1}+G_{2}$ denote the join [39] of two fuzzy graphs $G_{i}=\left(V_{i}, \sigma_{i}, \mu_{i}\right), i=1,2$ is defined as a fuzzy graph $G=\left(V_{1} \cup V_{2}, \sigma_{1}+\sigma_{2}, \mu_{1}+\mu_{2}\right)$ where $E=E_{1} \cup E_{2} \cup E^{\prime}$ and $E^{\prime}$ is the set of all edges joining vertices of $V_{1}$ with the vertices of $V_{2}$, and we assume that $V_{1} \cap V_{2}=\emptyset$. Fuzzy sets $\sigma_{1}+\sigma_{2}$ and $\mu_{1}+\mu_{2}$ are defined as $\left(\sigma_{1}+\sigma_{2}\right)(u)=\left(\sigma_{1} \cup \sigma_{2}\right)(u)$ and $\forall u \in V_{1} \cup V_{2} ;\left(\mu_{1}+\mu_{2}\right)(u v)=\left(\mu_{1} \cup \mu_{2}\right)(u v)$ if $u v \in E_{1} \cup E_{2}$ and $\left(\mu_{1}+\mu_{2}\right)(u v)=\sigma_{1}(u) \wedge \sigma_{2}(v)$ if $u v \in E^{\prime}$.

The classical paper [49] of Nordhaus and Gaddum established the inequalities for the chromatic numbers of a graph $G=(V, E)$ and its complement $\bar{G}$. We are concerned with analogous inequalities involving domination parameters in graphs. We begin with a brief overview of Nordhaus-Gaddum (NG) inequalities for several domination-related parameters. For each generic invariant $\mu$ of a graph $G$, let $\mu=\mu(G)$ and $\bar{\mu}=\mu(\bar{G})$. 
Inequalities on $\mu+\bar{\mu}$ and $\mu \cdot \bar{\mu}$ exist in the literature for only a few of the many domination-related parameters and most of these results are of the additive form. In 1972 Jaeger and Payan [26] published the first NG results involving domination. Cockayne and Hedetniemi [18] sharpened the upper bound for the sum. Laskar and Peters [33] improved this bound for the case when both $G$ and $\bar{G}$ are connected. A much improved bound was established for the case when neither $G$ nor $\bar{G}$ has isolated nodes by Bollobás and Cockayne [14] and by Joseph and Arumugam [27] independently.

Remark 2.1. For the sake of simplicity, we do sometimes

- writing $x y$ instead of $\{x, y\}$.

- calling $x$ both vertex and node.

- calling $x y$ both edge and arc.

- writing Cartesian product both $\square$ and $\times$.

- saying $\sigma(x)$ and $\mu(x y)$ with different literature, e.g. value, weight, membership value and etc.

\section{Main Results}

In this section, we provide the main results. We first briefly illustrate our opinion.

The terms "dominating set", and "domination number" of a graph $\mathrm{G}=(\mathrm{V}, \mathrm{E})$ were first defined by $\mathrm{O}$. Ore in 1962. A subset $A \subseteq V$ is a dominating set for $\mathrm{G}$ if each element of $\mathrm{V}$ is either in $\mathrm{A}$, or is adjacent to an element of $\mathrm{A}$. The domination number $\gamma(G)$, which is the most commonly used domination number, is the minimum cardinality among all dominating sets of $\mathrm{G}$.

More than 1200 papers already published on domination in graphs. Without a doubt, the literature on this subject is growing rapidly, and a considerable amount of work has been dedicated to find different bounds for the domination numbers of graphs. In Analogous to them in fuzzy graphs are existed many variations of concepts of dominations as mentioned some results on introduction. a few researchers defined, sometimes redefined, and studied other domination variations: such as connected domination, strong domination, total domination, (1,2)-vertex domination, 2-domination, domination and etc. However, from practical point of view, it was necessary to define other types of dominations. Most of these new variations required the dominating set to have additional properties such as: being as independent set, inducing a connected subgraph, or inducing a clique. These properties were reflected in their names as an adjective: independent domination, connected domination, and clique domination, respectively.

The $\alpha$-strong domination number of a fuzzy graph is defined in a classic way, Definition (3.1), (3.3), (3.4).

Definition 3.1. Let $G=(\sigma, \mu)$ be a fuzzy graph on $V$. Let $x, y \in V$. We say that $x$ dominates $y$ in $G$ as $\alpha$-strong if the arc $\{x, y\}$ is $\alpha$-strong.

Example 3.2. By attention to fuzzy graph In Figure (1), the $\operatorname{arcs} v_{2} v_{5}, v_{2} v_{4}, v_{3} v_{4}$ and $v_{1} v_{3}$ are $\alpha$-strong and the $\operatorname{arcs} v_{1} v_{4}, v_{1} v_{2}$ and $v_{4} v_{5}$ are not $\alpha$-strong.

Definition 3.3. A subset $S$ of $V$ is called a $\alpha$-strong dominating set in $G$ if for every $v \notin S$, there exists $u \in S$ such that $u$ dominates $v$.

Definition 3.4. Let $S$ be the set of all $\alpha$-strong dominating sets in $G$, the vertex domination number of $G$ is defined as $\min _{D \in S}\left[\Sigma_{u \in D}\left(\sigma(u)+\frac{d_{s}(u)}{d(u)}\right)\right]$ and it is denoted 
by $\gamma_{\mathbf{v}}(\mathbf{G})$. If $d(u)=0$, then we consider $\frac{d_{s}(u)}{d(u)}$ equal with 0 . The $\alpha$-strong dominating set that is correspond to $\gamma_{v}(G)$ is called by vertex dominating set. We also say $\Sigma_{u \in D}\left(\sigma(u)+\frac{d_{s}(u)}{d(u)}\right)$, vertex weight of $D$, for every $D \in S$ and it is denoted by $\mathbf{w}_{\mathbf{v}}(\mathbf{D})$.

Example 3.5. By attention to fuzzy graph In Figure (1), the set $\left\{v_{2}, v_{3}\right\}$ is the $\alpha$-strong dominating set. This set is also vertex dominating set in fuzzy graph $G$. Hence $\gamma_{v}(G)=1.75+0.9+0.7=3.35$. So $\gamma_{v}(G)=3.35$.

Theorem 3.6. [38] If $G$ is a complete fuzzy graph, then all arcs are strong.

Theorem 3.7. [38] If $G$ is a complete bipartite fuzzy graph, then all arcs are strong.

Remark 3.8. If $\mathrm{G}$ is a complete fuzzy graph, then all arcs are $\alpha$-strong.

Remark 3.9. If $\mathrm{G}$ is a complete bipartite fuzzy graph, then all arcs are $\alpha$-strong.

It is well known and generally accepted that the problem of determining the domination number of an arbitrary graph is a difficult one. Because of this, researchers have turned their attention to the study of classes of graphs for which the domination problem can be solved in polynomial time.

We determine vertex domination number for several classes of fuzzy graphs consists of complete fuzzy graph, Proposition (3.10), empty fuzzy graph, Proposition (3.11), star fuzzy graph, Proposition (3.13), complete bipartite fuzzy graph, Proposition (3.14).

Proposition 3.10 (Complete fuzzy graph). If $G=(V, \sigma, \mu)$ is a complete fuzzy graph, then $\gamma_{v}(G)=\min _{u \in V}(\sigma(u))+1$.

Proof. Since $G$ is a complete fuzzy graph, all arcs are $\alpha$-strong by Remark (3.8) and each node is incident to all other nodes. Hence $D=\{u\}$ is a $\alpha$-strong dominating set and $d_{s}(u)=d(u)$ for each $u \in V$ Hence the result follows.

Proposition 3.11 (Empty fuzzy graph). Let $G=(V, \sigma, \mu)$ be a fuzzy graph. Then $\gamma_{v}(G)=p$, if $G$ be edgeless, i.e. $G=\bar{K}_{n}$.

Proof. Since $G$ is edgeless, Hence $V$ is only $\alpha$-strong dominating set in $G$ and all arcs are not $\alpha$-strong. so we have $\gamma_{v}(G)=\min _{D \in S}\left[\Sigma_{u \in D} \sigma(u)\right]=\Sigma_{u \in v} \sigma(u)=p$ by Definition (3.4). so we can write $\gamma_{v}\left(\bar{K}_{n}\right)=p$ by our notations.

It is interesting

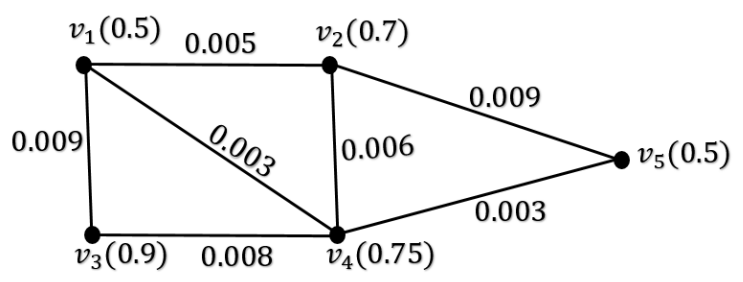
to note the converse of Proposition (3.11) that does not hold.

Example 3.12. We show the converse of Proposition (3.11) does not hold. For this purpose, Let $V=\left\{v_{1}, v_{2}, v_{3}, v_{4}, v_{5}\right\}$. We define $\sigma$ on $V$ by $\sigma: V \rightarrow[0,1]$ such that

Figure 1. Vertex domination

$$
\sigma\left(v_{1}\right)=0.5, \sigma\left(v_{2}\right)=0.7, \sigma\left(v_{3}\right)=0.9, \sigma\left(v_{4}\right)=0.75, \sigma\left(v_{5}\right)=0.5
$$

Now, The function $\mu: V \times V \rightarrow[0,1]$ is defined by

$$
\mu\left(v_{1} v_{2}\right)=0.005, \mu\left(v_{1} v_{4}\right)=0.003, \mu\left(v_{1} v_{3}\right)=0.009, \mu\left(v_{2} v_{4}\right)=0.006, \mu\left(v_{2} v_{5}\right)=0.009,
$$

$\mu\left(v_{3} v_{4}\right)=0.008, \mu\left(v_{4} v_{5}\right)=0.003$ such that $\forall u, v \in V, \mu(u, v) \leq \sigma(u) \wedge \sigma(v)$. Finally, Let $V, \sigma$, and $\mu$ be the vertices, value of vertices and value of edges respectively. In other words, By attention to fuzzy graph In Figure (1), the $\operatorname{arcs} v_{2} v_{5}, v_{2} v_{4}, v_{3} v_{4}$ and $v_{1} v_{3}$ are $\alpha$-strong and the $\operatorname{arcs} v_{1} v_{4}, v_{1} v_{2}$ and $v_{4} v_{5}$ are not $\alpha$-strong. So the set $\left\{v_{2}, v_{3}\right\}$ is the $\alpha$-strong dominating set. This set is also vertex dominating set in fuzzy graph $G$. Hence $\gamma_{v}(G)=1.75+0.9+0.7=3.35=\Sigma_{u \in v} \sigma(u)=p$. So $G \neq \bar{K}_{5}$ but $\gamma_{v}(G)=p$. 
Proposition 3.13 (Star fuzzy graph). Let $G$ be a star fuzzy graph. Then $G=K_{1, \sigma}$ and $\gamma_{v}\left(K_{1, \sigma}\right)=\sigma(u)+1$ where $u$ is center of $G$.

Proof. Let $G$ be the star fuzzy graph with $V=\left\{u, v_{1}, v_{2}, \cdots, v_{n}\right\}$ such that $u$ and $v_{i}$ are center and leaves of $G$, for $1 \leq i \leq n$ respectively. So $G^{*}=K_{1, n}^{*}$ is underlying crisp graph of $G$. $\{u\}$ is vertex dominating set in $G$ and all arcs are $\alpha$-strong by Remark (3.9) and due to $G$ is bipartite fuzzy graph. Hence the result follows.

Proposition 3.14 (Bipartite fuzzy graph). Let $G$ be the bipartite fuzzy graph which is not star fuzzy graph. Then $G=K_{\sigma_{1}, \sigma_{2}}$ and $\gamma_{v}\left(K_{\sigma_{1}, \sigma_{2}}\right)=\min _{u \in V_{1}, v \in V_{2}}(\sigma(u)+\sigma(v))+2$.

Proof. Let $G \neq K_{1, \sigma}$ be bipartite fuzzy graph. Then both of $V_{1}$ and $V_{2}$ include more than one vertex. In $K_{\sigma_{1}, \sigma_{2}}$, all arcs are $\alpha$-strong by Remark (3.9). Also each node in $V_{1}$ is dominated as $\alpha$-strong with all nodes in $V_{2}$ and conversely. Hence in $K_{\sigma_{1}, \sigma_{2}}$, the $\alpha$-strong dominating sets are $V_{1}$ and $V_{2}$ and any set containing 2 nodes, one in $V_{1}$ and other in $V_{2}$. Hence $\gamma_{v}\left(K_{\sigma_{1}, \sigma_{2}}\right)=\min _{u \in V_{1}, v \in V_{2}}(\sigma(u)+\sigma(v))+2$. So the theorem is proved.

We give an upper bound for the vertex domination number of fuzzy graphs, Proposition (3.15).

Proposition 3.15. For any fuzzy graph $G=(V, \sigma, \mu)$, We have $\gamma_{v} \leq p$.

Proof. $\gamma_{v}\left(\bar{K}_{n}\right)=p$ by Theorem (3.11). So the result follows.

For the vertex domination number $\gamma_{v}$ the following theorem gives a Nordhaus-Gaddum type result.

For any fuzzy graph the Nordhaus-Gaddum(NG)'result holds, Theorem (3.16).

Theorem 3.16. For any fuzzy graph $G=(V, \sigma, \mu)$, The Nordhaus-Gaddum result holds. In other words, we have $\gamma_{v}+\bar{\gamma}_{v} \leq 2 p$.

Proof. $G$ is fuzzy graph. So $\bar{G}$ is also fuzzy graph. We implement Theorem (3.15) on $G$ and $\bar{G}$. Then $\gamma_{v} \leq p$ and $\overline{\gamma_{v}} \leq p$. Hence $\gamma_{v}+\overline{\gamma_{v}} \leq 2 p$. So the theorem is proved.

The following theorems on dominating sets in graphs are the first results about domination and were presented by Ore in his book Theory of Graphs [69].

Definition 3.17 ( [47]). A $\alpha$-strong dominating set $D$ is called a minimal $\alpha$-strong dominating set if no proper subset of $\mathrm{D}$ is a $\alpha$-strong dominating set.

Theorem 3.18 ( [47]). Let $G$ be a fuzzy graph without isolated nodes. If $D$ is a minimal $\alpha$-strong dominating set then $V-D$ is a $\alpha$-strong dominating set.

Finding a domatic partition of size 1 is trivial and finding a domatic partition of size 2 (or establishing that none exists) is easy but finding a maximum-size domatic partition (i.e., the domatic number), is computationally hard. Finding domatic partition of size two in fuzzy graph $G$ of order $n \geq 2$ is easy by the following.

Theorem 3.19 ( [47]). Every connected graph $G$ of order $n \geq 2$ has a $\alpha$-strong dominating set $D$ whose complement $V-D$ is also a $\alpha$-strong dominating set.

We improve upper bound for the vertex domination number of fuzzy graphs without isolated nodes, Theorem (3.20).

Theorem 3.20. For any fuzzy graph $G=(V, \sigma, \mu)$ without isolated nodes, We have $\gamma_{v} \leq \frac{p}{2}$. 
Proof. Let $D$ be a minimal dominating set of $G$. Then by Theorem (3.19), V-D is a $\alpha$-strong dominating set of $G$. Then $\gamma_{v}(G) \leq w_{v}(D)$ and $\gamma_{v}(G) \leq w_{v}(V-D)$.

Therefore $2 \gamma_{v}(G) \leq w_{v}(D)+w_{v}(V-D) \leq p$ which implies $\gamma_{v} \leq \frac{p}{2}$. Hence the proof is completed.

We also improve Nordhaus-Gaddum(NG)'result for fuzzy graphs without isolated nodes, Corollary (3.21).

Corollary 3.21. Let $G$ be a fuzzy graph such that both $G$ and $\bar{G}$ have no isolated nodes. Then $\gamma_{v}+\overline{\gamma_{v}} \leq p$, where $\overline{\gamma_{v}}$ is the vertex domination number of $\bar{G}$. Moreover, equality holds if and only if $\gamma_{v}=\bar{\gamma}_{v}=\frac{p}{2}$.

Proof. By the Implement of Theorem (3.20) on $G$ and $\bar{G}$, we have $\gamma_{v}(G)=\gamma_{v} \leq \frac{p}{2}$, and $\gamma_{v}(\bar{G})=\bar{\gamma}_{v}(G)=\overline{\gamma_{v}} \leq \frac{p}{2}$. So $\gamma_{v}+\bar{\gamma}_{v} \leq \frac{p}{2}+\frac{p}{2}=p$. Hence $\gamma_{v}+\overline{\gamma_{v}} \leq p$.

Suppose $\gamma_{v}=\overline{\gamma_{v}}=\frac{p}{2}$, then obviously $\gamma_{v}+\overline{\gamma_{v}}=p$. Conversely, suppose $\gamma_{v}+\overline{\gamma_{v}} \leq p$. Then we have $\gamma_{v} \leq \frac{p}{2}$ and $\overline{\gamma_{v}} \leq \frac{p}{2}$. If either $\gamma_{v}<\frac{p}{2}$ or $\overline{\gamma_{v}}<\frac{p}{2}$, then $\gamma_{v}+\overline{\gamma_{v}}<p$, which is a contradiction. Hence the only possibility case is $\gamma_{v}=\overline{\gamma_{v}}=\frac{p}{2}$.

Remark 3.22. Note that when we use the definition of domination number in $[13,14,15]$, Theorem (3.20) and Corollary (3.21) are hold.

Proposition 3.23. Let $G=(V, \sigma, \mu)$ be a fuzzy graph. If all arcs have equal value, the $G$ has no $\alpha$-strong edge.

Proof. Obviously the result is hold by using Definition (3.1).

We give the relationship between $M$-strong arcs and $\alpha$-strong arcs, Corollary (3.24).

Corollary 3.24. Let $G=(V, \sigma, \mu)$ be a fuzzy graph. If all arcs are $M$-strong, the $G$ has no $\alpha$-strong edge.

Proof. Obviously the result is hold by using Proposition (3.23).

Figure 2. $M$-strong arcs and $\alpha$-strong arcs
The following example illustrates this concept.

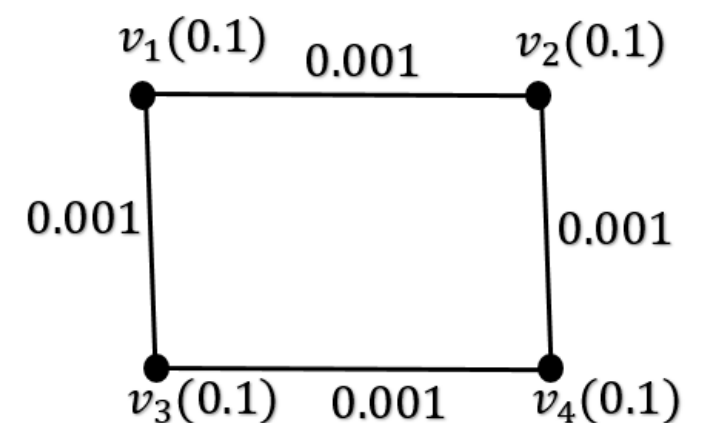

Example 3.25.

In Figure (2), all arcs are $M$-strong but there is no $\alpha$-strong arcs in this fuzzy graph. Obviously this result is hold by using Definition (3.3).

We give a necessary and sufficient condition for vertex domination which is half of order, In fact fuzzy graphs with vertex

domination which is half of order is characterized in the special case, Theorem (3.26).

Theorem 3.26. In any fuzzy graph $G=(V, \sigma, \mu)$ such that values of nodes are equal and all arcs have same value, i.e. for $\forall u_{i}, u_{j} \in V$ and $\forall e_{i}, e_{j} \in E$, we have $\sigma\left(u_{i}\right)=\sigma\left(u_{j}\right)$ and $\mu\left(e_{i}\right)=\mu\left(e_{j}\right) . \gamma_{v}=\frac{p}{2}$ if and only if For any vertex dominating set $D$ in $G$, we have $|D|=\frac{n}{2}$.

Proof. Suppose $D$ has the conditions. $d_{s}(D)=0$ by Proposition (3.23). So $\gamma_{v}(G)=\Sigma_{u \in D} \sigma(u)$ by using Definition (3.4). Since values of nodes are equal and $|D|=\frac{n}{2}$, we have $\gamma_{v}(G)=\Sigma_{u \in D} \sigma(u)=\frac{n}{2} \sigma(u)=\frac{1}{2}(n \sigma(u))=\frac{1}{2}\left(\Sigma_{u \in V} \sigma(u)\right)=\frac{1}{2}(p)=\frac{p}{2}$. Hence the result is hold in this case. 
Conversely, Suppose $\gamma_{v}=\frac{p}{2}$. Let $D=\left\{u_{1}, u_{2}, \cdots, u_{n}\right\}$ be a vertex dominating set. $d_{s}(D)=0$ by Proposition (3.23). So $\gamma_{v}(G)=\Sigma_{u \in D} \sigma(u)$ by using Definition (3.4). Since $\gamma_{v}(G)=W_{v}(D)$, we have $\gamma_{v}=\frac{p}{2}=\frac{1}{2}\left(\Sigma_{u \in V} \sigma(u)\right)=\Sigma_{u \in D} \sigma(u)$. Suppose $n^{\prime} \neq \frac{n}{2}$. so $\left.\sum_{i=1}^{n^{\prime \prime}} \sigma\left(v_{i}\right)\right)=0$ which is a contradiction with $\forall u_{i} \in V, \sigma\left(u_{i}\right)>0$. Hence $n^{\prime}=\frac{n}{2}$, i.e. $|D|=n^{\prime}=\frac{n}{2}$. So the result is hold in this case.

The vertex domination of union of two fuzzy graphs is studied, Proposition (3.27).

Proposition 3.27. Let $G_{1}$ and $G_{2}$ be fuzzy graphs. The vertex dominating set of $G_{1} \cup G_{2}$ is $D=D_{1} \cup D_{2}$ such that $D_{1}$ and $D_{2}$ are the vertex dominating set of $G_{1}$ and $G_{2}$ respectively. Moreover, $\gamma_{v}\left(G_{1} \cup G_{2}\right)=\gamma_{v}\left(G_{1}\right)+\gamma_{v}\left(G_{2}\right)$.

Proof. Obviously the result is hold by using Definition of union of two fuzzy graphs.

Also the vertex domination of union of fuzzy graphs Family is discussed, Corollary (3.28).

Corollary 3.28. Let $G_{1}, G_{2}, \cdots, G_{n}$ be fuzzy graphs. The vertex dominating set of $\cup_{i=1}^{n} G_{i}$ is $D=\cup_{i=1}^{n} D_{i}$ such that $D_{i}$ is the vertex dominating set of $G_{i}$. Moreover, $\gamma_{v}\left(\cup_{i=1}^{n} G_{i}\right)=\sum_{i=1}^{n} \gamma_{v}\left(G_{i}\right)$.

Proof. Obviously the result is hold by using proposition (3.27).

The concepts of both monotone increasing fuzzy graph property, Definition (3.29), and monotone decreasing fuzzy graph property, Definition (3.31), are introduced.

Definition 3.29. We call a fuzzy graph property $P$ monotone increasing if $G \in P$ implies $G+e \in P$, i.e., adding an edge e to a fuzzy graph $G$ does not destroy the property.

Example 3.30. Connectivity and Hamiltonicity are monotone increasing properties. A monotone increasing property is nontrivial if the empty graph $\bar{K}_{n} \notin P$ and the complete graph $K_{n} \in P$.

Definition 3.31. A fuzzy graph property is monotone decreasing if $G \in P$ implies $G-e \in P$, i.e., removing an edge from a graph does not destroy the property.

Example 3.32. Properties of a fuzzy graph not being connected or being planar are examples of monotone decreasing fuzzy graph properties.

Remark 3.33. Obviously, a fuzzy graph property $\mathrm{P}$ is monotone increasing if and only if its complement is monotone decreasing. Clearly not all fuzzy graph properties are monotone. For example having at least half of the vertices having a given fixed degree $d$ is not monotone.

Let $\gamma(G)$ denote the domination number of a simple graph $G$. Then Vizing (1963) [17]conjectured that $\gamma(G) \gamma(H) \leq \gamma(G \times H)$, where $G \times H$ is the graph product. While the full conjecture remains open, Clark and Suen (2000) [23] have proved the looser result $\gamma(G) \gamma(H) \leq 2 \gamma(G \times H)$.

Vizing stated the still open conjecture:

Conjecture (Vizing [17]). For all graphs $G$ and $H, \gamma(G) \gamma(H) \leq \gamma(G \times H)$. The result in relation with vizing's conjecture by using $\alpha$-strong arc and monotone decreasing fuzzy graph property is determined, Theorem (3.34).

Theorem 3.34. The vizing's conjecture is monotone decreasing property in fuzzy graph $G$, if the edge e be $\alpha$-strong and $\gamma_{v}(G-e)=\gamma_{v}(G)$. 
Proof. The fuzzy graph $(G-e) \times H$ is the spanning fuzzy subgraph of $G \times H$, for all fuzzy graph $H$. So $\gamma_{v}((G-e) \times H) \geq \gamma_{v}(G \times H) \geq \gamma_{v}(G) \gamma_{v}(H)=\gamma_{v}(G-e) \gamma_{v}(H)$. Hence vizing's conjecture is also hold for $G-e$. Then the result follows.

Some results in relation with vizing's conjecture by using $\alpha$-strong arc and spanning fuzzy subgraph is studied, Corollary (3.35).

Corollary 3.35. Suppose the vizing's conjecture is hold for $G$. Let $K$ be the spanning fuzzy subgraph of $G$ such that $\gamma_{v}(K)=\gamma_{v}(G)$. Then the vizing's conjecture is hold for $K$.

Proof. The fuzzy graph $K \times H$ is the spanning fuzzy subgraph of $G \times H$, for all fuzzy graph $H$. So $\gamma_{v}(K \times H) \geq \gamma_{v}(G \times H) \geq \gamma_{v}(G) \gamma_{v}(H)=\gamma_{v}(K) \gamma_{v}(H)$. Hence the vizing's conjecture is also hold for $K$. So the result follows.

The vertex domination of join of two fuzzy graphs is studied, Proposition (3.36).

Proposition 3.36. Let $G_{1}$ and $G_{2}$ be fuzzy graphs. The vertex dominating set of $G_{1} \otimes G_{2}$ is $D=D_{1} \cup D_{2}$ such that $D_{1}$ and $D_{2}$ are the vertex dominating set of $G_{1}$ and $G_{2}$ respectively. Moreover, $\gamma_{v}\left(G_{1} \otimes G_{2}\right)=\gamma_{v}\left(G_{1}\right)+\gamma_{v}\left(G_{2}\right)$.

Proof. Obviously the result is hold by using Definition of join of two fuzzy graphs and Corollary (3.24) which state in this case, $M$-strong arcs between two fuzzy graphs is not $\alpha$-strong which is weak arc changing strength of connectedness of $G$.

Also the vertex domination of join of fuzzy graphs Family is discussed, Corollary $(3.37)$.

Corollary 3.37. Let $G_{1}, G_{2}, \cdots, G_{n}$ be fuzzy graphs. The vertex dominating set of $\otimes_{i=1}^{n} G_{i}$ is $D=\otimes_{i=1}^{n} D_{i}$ such that $D_{i}$ is the vertex dominating set of $G_{i}$. Moreover, $\gamma_{v}\left(\otimes_{i=1}^{n} G_{i}\right)=\sum_{i=1}^{n} \gamma_{v}\left(G_{i}\right)$.

Proof. Obviously the result is hold by using proposition (3.36).

Gravier and Khelladi [22] conjecture a Vizing-like inequality for the domination number of the cross product of graphs.

Gravier and Khelladi stated the still open conjecture:

Conjecture (Gravier and Khelladi [22]). For all graphs $G$ and $H$,

$$
\gamma(G) \gamma(H) \leq 2 \gamma(G \otimes H) .
$$

The result in relation with Gravier and Khelladi's conjecture by using $\alpha$-strong arc and monotone decreasing fuzzy graph property is determined, Theorem (3.38).

Theorem 3.38. The Gravier and Khelladi's conjecture is monotone decreasing property in fuzzy graph $G$, if the edge e be $\alpha$-strong and $\gamma_{v}(G-e)=\gamma_{v}(G)$.

Proof. The fuzzy graph $(G-e) \times H$ is the spanning fuzzy subgraph of $G \times H$, for all fuzzy graph $H$. So $\gamma_{v}((G-e) \times H) \geq \gamma_{v}(G \times H) \geq \gamma_{v}(G) \gamma_{v}(H)=\gamma_{v}(G-e) \gamma_{v}(H)$. Hence Gravier and Khelladi's conjecture is also hold for $G-e$. Then the result follows.

We conclude this section with Some result in relation with Gravier and Khelladi's conjecture by using $\alpha$-strong arc and spanning fuzzy subgraph is studied, Corollary (3.39).

506 
Corollary 3.39. Suppose the Gravier and Khelladi's conjecture is hold for G. Let $K$ be ${ }_{517}$ the spanning fuzzy subgraph of $G$ such that $\gamma_{v}(K)=\gamma_{v}(G)$. Then the Gravier and Khelladi's conjecture is hold for $K$.

Proof. The fuzzy graph $K \times H$ is the spanning fuzzy subgraph of $G \times H$, for all fuzzy graph $H$. So $\gamma_{v}(K \otimes H) \geq \gamma_{v}(G \otimes H) \geq \gamma_{v}(G) \gamma_{v}(H)=\gamma_{v}(K) \gamma_{v}(H)$. Hence the Gravier and Khelladi's conjecture is also hold for $K$. So the result follows.

\section{Practical applications}

In this Section, We give 9 practical applications in relation with these concepts.

Domination is a rapidly developing area of research in graph theory. The concept of domination

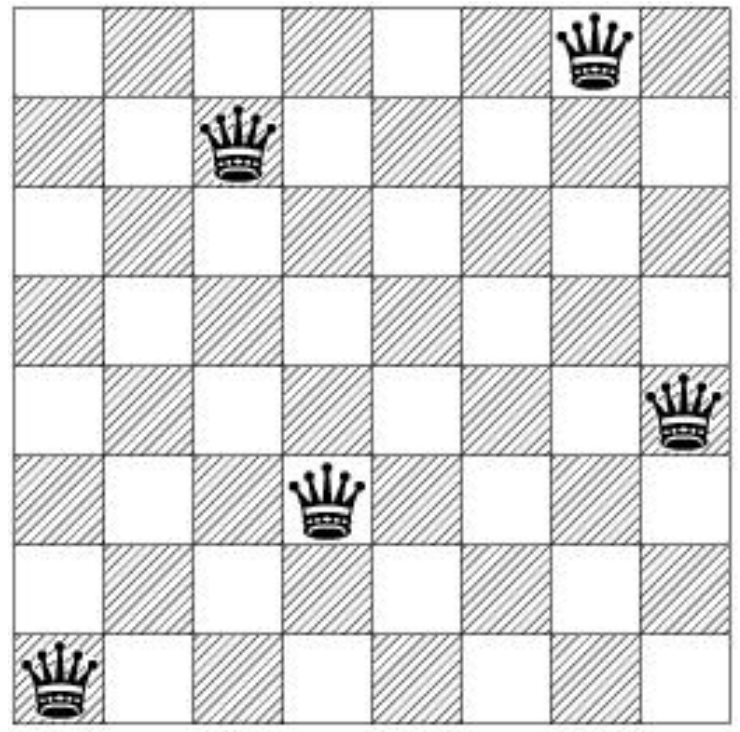
has existed for a long time and early discussions on the topic can be found in works of Ore and Berge . The summary of the literature shows the following wide-known problems, which are considered among the earliest applications for dominating sets.

\section{Queens Problem}

This problem was mentioned by Ore. According to the rules of chess a queen can, in one move, advance any number of squares horizontally, diagonally, or vertically (assuming that no other chess figure is on its way). How to place a minimum number of queens on a chessboard so that each square is controlled by at least one queen? See one of the solutions in (Fig. 3). For fuzzification of this problem, types of square (based on sensitive place in game of chess, chess pieces) and types of connection can be assigned by different values. So the question is changed to this. How to place a number of queens on a chessboard so that each square is controlled by at least one queen based on values on queens and ratio of total of values of adjacent $\alpha$-strong connections to total of values of adjacent connections?

\section{Locating Radar Stations Problem}

The problem was discussed by Berge. A number of strategic locations are to be kept under surveillance. The goal is to locate a radar for the surveillance at as few of these locations as possible. How a set of locations in which the radar stations are to be placed can be determined? For fuzzification of this problem, types of radar stations (based on power of them) and types of connection with locations can be assigned by different values. So the question is changed to this. How a set of locations in which the radar stations are to be placed can be determined based on values on radar stations and ratio of total of values of adjacent $\alpha$-strong connections to total of values of adjacent connections?

\section{Problem of Communications in a Network}

Suppose that there is a network of cities with communication links. How to set up transmitting stations at some of the cities so that every city can receive a message from at least one of the transmitting stations? This problem was discussed in detail by Liu. For fuzzification of this problem, types of cities (based on population, structure) and 
types of connection with cities can be assigned by different values. So the question is changed to this. How to set up transmitting stations at some of the cities so that every city can receive a message from at least one of the transmitting stations based on values on cities and ratio of total of values of adjacent $\alpha$-strong connections to total of values of adjacent connections?

\section{Nuclear Power Plants Problem}

A similar known problem is a nuclear power plants problem. There are various locations and an arc can be drawn from location $\mathrm{x}$ to location $\mathrm{y}$ if it is possible for a watchman stationed at $\mathrm{x}$ to observe a warning light located at $\mathrm{y}$. How many guards are needed to observe all of the warning lights, and where should they be located? For fuzzification of this problem, types of guards (based on abilities) and types of connection with guards can be assigned by different values. So the question is changed to this. How many guards are needed to observe all of the warning lights, and where should they be located based on values on guards and ratio of total of values of adjacent $\alpha$-strong connections to total of values of adjacent connections?

At present, domination is considered to be one of the fundamental concepts in graph theory and its various applications to ad hoc networks, biological networks, distributed computing, social networks and web graphs partly explain the increased interest. Such applications usually aim to select a subset of nodes that will provide some definite service such that every node in the network is ?close? to some node in the subset. The following examples show when the concept of domination can be applied in modelling real-life problems.

\section{Modelling Biological Networks}

Using graph theory as a modelling tool in biological networks allows the utilization of the most graphical invariants in such a way that it is possible to identify secondary RNA (Ribonucleic acid) motifs numerically. Those graphical invariants are variations of the domination number of a graph. The results of the research carried out show that the variations of the domination number can be used for correctly distinguishing among the trees that represent native structures and those that are not likely candidates to represent RNA. For fuzzification of this problem, types of location (based on advantages) and types of connection with locations can be assigned by different values. So the question is based on based on values on locations and ratio of total of values of adjacent $\alpha$-strong connections to total of values of adjacent connections?

\section{Modelling Social Networks}

Dominating sets can be used in modelling social networks and studying the dynamics of relations among numerous individuals in different domains. A social network is a social structure made of individuals (or groups of individuals), which are connected by one or more specific types of interdependency. The choice of initial sets of target individuals is an important problem in the theory of social networks. In the work of Kelleher and Cozzens, social networks are modelled in terms of graph theory and it was shown that some of these sets can be found by using the properties of dominating sets in graphs. For fuzzification of this problem, types of people (based on abilities) and types of connection with people can be assigned by different values. So the question is based on based on values on people and ratio of total of values of adjacent $\alpha$-strong connections to total of values of adjacent connections?

\section{Facility Location Problems}

The dominating sets in graphs are natural models for facility location problems in operational research. Facility location problems are concerned with the location of one or more facilities in a way that optimizes a certain objective such as minimizing transportation cost, providing equitable service to customers and capturing the largest market share. For fuzzification of this problem, types of location (based on advantages) and types of connection with locations can be assigned by different values. So the 
question is based on based on values on locations and ratio of total of values of adjacent $\alpha$-strong connections to total of values of adjacent connections?

\section{Coding Theory}

The concept of domination is also applied in coding theory as discussed by Kalbfleisch, Stanton and Horton and Cockayne and Hedetniemi. If one defines a graph, the vertices of which are the n-dimensional vectors with coordinates chosen from $\{1, \cdots, p\}, p>1$, and two vertices are adjacent if they differ in one coordinate, then the sets of vectors which are $(n, p)$-covering sets, single error correcting codes, or perfect covering sets are all dominating sets of the graph with determined additional properties. For fuzzification of this problem, types of codes (based on types of words, different words, same words) and types of connection with codes can be assigned by different values. So the question is based on based on values on codes and ratio of total of values of adjacent $\alpha$-strong connections to total of values of adjacent connections?

\section{Multiple Domination Problems}

An important role is played by multiple domination. Multiple domination can be used to construct hierarchical overlay networks in peer-to-peer applications for more efficient index searching. The hierarchical overlay networks usually serve as distributed databases for index searching, e.g. in modern file sharing and instant messaging computer network applications. Dominating sets of several kinds are used for balancing efficiency and fault tolerance as well as in the distributed construction of minimum spanning trees. Another good example of direct, important and quickly developing applications of multiple domination in modern computer networks is a wireless sensor network. A wireless sensor net- work (WSN) usually consists of up to several hundred small autonomous devices to measure some physical parameters. Each device contains a processing unit and a limited memory as well as a radio transmitter and a receiver to be able to communicate with its neighbors. Also, it contains a limited power battery and is constrained in energy consumption. There is a base station, which is a special sensor node used as a sink to collect information gathered by other sensor nodes and to provide a connection between the WSN and a usual network. A routing algorithm allows the sensor nodes to self-organize into a WSN. As stated, an important goal in WSN design is to maximize the functional lifetime of a sensor network by using energy efficient distributed algorithms, networking and routing techniques. To maximize the functional lifetime, it is important to select some sensor nodes to behave as a backbone set to support routing communications. The backbone set can be considered as a dominating set in the corresponding graph. Dominating sets of several different kinds have proved to be useful and effective for modelling backbone sets. In the recent literature, particular attention has been paid to construction of k-connected kdominating sets in WSNs, and several probabilistic and deterministic approaches have been proposed and analyzed. The backbone set of sensor nodes should be selected as small as possible and, on the other hand, it should guarantee high efficiency and reliability of networking and communications. This trade-off requires construction of multiple dominating sets providing energy efficient and reliable data dissemination and communication protocols. For fuzzification of this problem, types of sensor nodes (based on advantages) and types of connection with sensor nodes can be assigned by different values. So the question is based on based on values on sensor nodes and ratio of total of values of adjacent $\alpha$-strong connections to total of values of adjacent connections?

A homogeneous WSN consists of wireless sensor devices of the same kind. All the devices have the same set of limited resources and, originally, no hierarchy is imposed on the network structure and communications. In a network of this kind, the only special sensor node is a base station. For all the other nodes, it is necessary to construct and switch the backbone sets and communications efficiently so that all the network nodes stay in operation as long as possible. Therefore, in this case, it is important to be 
able to construct and switch dominating sets and route communications uniformly and efficiently with respect to the energy consumption of each particular sensor node. This has to be done to optimize the functional lifetime of the whole network.

Usually, a WSN is mathematically modelled as a unit or quasi-unit disk graph. These are the most natural and general graph models for a WSN. In a unit disk graph model, nodes correspond to sensor locations in the Euclidean plane and are assumed to have identical (unit) transmission ranges. An edge between two nodes means that they can communicate directly, i.e. the distance between them is at most one. A survey of known results on unit disk graphs, including algorithms for constructing dominating sets, can be found. A quasi-unit disk graph model takes into consideration possible transmission obstacles and is much closer to reality: we are sure to have an edge between two nodes if the distance between them is at most a parameter $d, 0<d<1$. If the distance between two nodes is in the range from $\mathrm{d}$ to 1 , the existence of an edge is not specified. A description of several more restricted geometric graph models for WSN design, e.g. the related neighborhood graph, Gabriel graph, Yao graph etc., can be found.

Domination is an area in graph theory with an extensive research activity. A book by Haynes, Hedetniemi and Slater on domination published in 1998 lists 1222 articles in this area.

\section{Conclusion}

Graph theory is one of the branches of modern mathematics having experienced a most impressive development in recent years. One of the most interesting problems in graph theory is that of Domination Theory. Nowadays domination theory ranks top among the most prominent areas of research in graph theory and combinatorics. The theory of domination has been the nucleus of research activity in graph theory in recent times. The fastest growing area within graph theory is a study of domination and related subset problems such independence, covering, matching, decomposition and labelling. Domination boasts a host of applications to social network theory, land surveying, game theory, interconnection network, parallel computing and image processing and so on. Today, this theory gained popularity and remains as a major area of research. At present, domination is considered to be one of the fundamental concepts in graph theory and its various applications to ad hoc networks, biological networks, distributed computing, social networks and web graphs partly explain the increased interest. More than 1200 papers already published on domination in graphs. Without a doubt, the literature on this subject is growing rapidly, and a considerable amount of work has been dedicated to find different bounds for the domination numbers of graphs. However, from practical point of view, it was necessary to define other types of dominations.

Most of these new variations required the dominating set to have additional properties.

\section{Acknowledgments}

We thank just about everybody. The author is highly thankful to the Editor-in-Chief and the referees for their valuable comments and suggestions for improving the paper.

\section{References}

1. M. Akram, Bipolar fuzzy graphs, Information Sciences, 181 (2011) 5548-5564.

2. M. Akram, Interval-valued fuzzy line graphs, Neural Computing and Applications, 21(2012) 145-150. 
3. M. Akram, Sheng-Gang Li and K.P. Shum, Antipodal bipolar fuzzy graphs, Italian Journal of Pure and Applied Mathematics, 31 (2013) 425-438.

4. S. Arumugam and R. Kala, Domination parameters of star graphs, Ars Combi. 44 (1996) 93-96.

5. D.B. Auer, F. Harary, J. Nieminen, and C.L. Suffel, Domination Alteration Sets in Graphs, Discrete Math. 47 (1983) 53-161.

6. C. Berge, Graphs and Hypergraphs, North Holland, Amsteddam, 1973.

7. C. Bezdek and S.K. Pal, Fuzzy Models for Pattern Recognition, IEEE Press, (1992).

8. Brešar, Boštjan; Dorbec, Paul; Goddard, Wayne; Hartnell, Bert L.; Henning, Michael A.; Klavžar, Sandi; Rall, Douglas F. (2012), Vizing's conjecture: a survey and recent results, Journal of Graph Theory 69 (1) 46-76.

9. P. Bhattacharya, Pattern Recognition Letter, 6 (1987) 297-302.

10. P. Bhattacharya, Some Remarks on fuzzy graphs, Pattern Recognition Letters 6 (1987) 297-302.

11. K.R. Bhutani, Pattern Recognition Letter, 9 (1989) 159-162.

12. K.R. Bhutani, A. Rosenfeld, Strong arcs in fuzzy graphs, Information Sciences, 152 (2003) 319-322.

13. K.R. Bhutani, J. Mordeson, and A. Rosenfeld, On degrees of end nodes and cut nodes in fuzzy graphs, Iranian Journal of Fuzzy Systems 11 (2004) 53-60.

14. B. Bollobás and E.J. Cockayne, unpublished.

15. K.R.Bhutani, A.Rosenfeld, Strong arcs in fuzzy graphs, Information Sciences 152 (2003) 319-322.

16. G. Chartrand, L. Lesniak, Graphs and Digraphs, 3rd ed., Wadsworth and Brooks Cole, Monterey, 1996.

17. W.E. Clark and S. Suen, An Inequality Related to Vizing's Conjecture, Electronic J. Combinatorics 71 (2000) 1-3, 2000.

18. E.J. Cockayne and S.T. Hedetniemi, Toward a theory of domination in graphs, Networks 7 (1977) 247-261.

19. E.J. Cockayne, and S.T. Hedetniemi, Towards the theory of domination in graphs, Networks 10 (1977) 247-261.

20. W.L. Craine, Fuzzy Sets and Systems, 68 (1994) 181-193.

21. M. Delgado, J.L. Verdegay and M.A. Vila, European J. Operations Research 22 (1985) 243-249.

22. S. Gravier, A. Khelladi, On the domination number of cross products of graphs, Discrete Mathematics, 145 (1995) 273-277.

23. Hartnell, B. and Rall, D. F. Domination in Cartesian Products: Vizing's Conjecture. In Domination in Graphs-Advanced Topics (Ed. T. W. Haynes, S. T. Hedetniemi, and P. J. Slater). New York: Dekker, (1998) 163-189. 
24. .W. Haynes, S.T. Hedetniemi, P.J. Slater, Fundamentals of Domination in Graphs, Marcel Dekker. New York, 1998.

25. Hedetniemi S.T. and Laskar.R.C., Topics on Domination, Vol. 48, North Holland, New York, 1990.

26. F. Jaeger and C. Payan, Relations du type Nordhaus-Gaddum pour le nombre d'absorption d'un graphe simple, C.R. Acad. Sci. Paris A 274 (1972) 728-730.

27. J.P. Joseph and S. Arumugam, A note on domination in graphs, lnternet. J. Management Systems, to appear.

28. Kaufmann.A., (1975), Introduction to the theory of Fuzzy Subsets, Academic Press, New york.

29. Anjaly Kishore and M.S.Sunitha, Strong chromatic number of fuzzy graphs, Annals of Pure and Applied Mathematics, 7(2) (2014) 52-60.

30. S. Klavžar, B. Zmazek, On a Vizing-like conjecture for direct product graphs, Discrete Mathematics, 156 (1996) 243-246.

31. G.J. Klir and Yuan Bo, Fuzzy Sets and Fuzzy Logic: Theory and Applications, PHI, (1997).

32. Kulli V.R., Theory of Domination in Graphs,Vishwa International Publications, 2010 .

33. R. Laskar and K. Peters, Vertex and edge domination parameters in graphs, Congr. Numer. 48 (1985) 291-305.

34. C.T. Leondes, Fuzzy Theory Systems: Techniques and Applications Vol. 1-4, Academic Press, (1999).

35. F.A. Lootsrna, Fuzzy Logic for Planning and Decision Making, Kluwer, (1997).

36. O. T. Manjusha and M. S. Sunitha, Notes on domination in fuzzy graphs, Journal of intelligent and Fuzzy Systems 27 (2014) 3205-3212.

37. O. T. Manjusha and M. S. Sunitha, Total domination in fuzzy graphs using strong arcs, Annals of Pure and Applied Mathematics 9 (1) (2014) 23-33.

38. S. Mathew and M.S. Sunitha, Node connectivity and arc connectivity of a fuzzy graph, Information Sciences 180 (2010) 519-531.

39. J.N. Mordeson, C.S. Peng, Operations on fuzzy graphs, Information Sciences 79 (1994) 159-170.

40. J.N. Mordeson and D.S. Malik, Fuzzy Commutative Algebra, World Scientific (1998).

41. J.N. Mordeson and P.S. Nair, Trees, Cycles and Cocycles of Fuzzy Graphs, Encyclopedia of Computer Science and Technology 39 Supplement 24 (1998) 301-322.

42. J.N. Mordeson, Pattern Recognition Letter, 14 (1993) 381-384.

43. J.N. Mordeson and C.S. Peng, Information Sciences, 79 (1994) 159-170.

44. J.N. Mordeson, P.S. Nair, Fuzzy Graphs and Fuzzy Hypergraphs, Physica-Verlag, 2000. 
45. J.N. Mordeson and P.S. Nair, Information Sciences, 90 (1996) 39-49.

46. J.N. Mordeson, Fuzzy line graphs, Pattern Recognition Letters 14 (1993) $381 ? 384$.

47. A. Nagoorgani and V. T. Chandrasekaran, Domination in fuzzy graph, Adv. in Fuzzy Sets and Systems I (1) (2006) 17-26.

48. Sk. Md. Abu Nayeem and M. Pal, Diameter constrained fuzzy minimum spanning tree problem, International Journal of Computational Intelligence Systems, 6(6) (2013) 1040-1051.

49. E.A. Nordhaus and J.W. Gaddum, On complementary graphs, Amer. Math. Monthly 63 (1956) 175-177.

50. O. Ore, Theory of Graphs, Amer. Math. Soc., Collog, Publish, 38, Providence,1962.

51. M.Pal and H.Rashmanlou, Irregular interval- valued fuzzy graphs, Annals of Pure and Applied Mathematics, 3(1) (2013) 56-66.

52. T. Pramanik, S. Samanta and M. Pal, Interval-valued fuzzy planar graphs, Int. J. Mach. Learn. and Cyber., DOI 10.1007/s13042-014-0284-7.

53. H. Rashmanlou and M. Pal, Antipodal interval-valued fuzzy graphs, International journal of Applications of Fuzzy Sets and Artificial Intelligence, 3 (2013) 107-130.

54. H. Rashmanlou and M. Pal, Isometry on interval-valued fuzzy graphs, International Journal of Fuzzy Mathematical Archive, 3 (2013) 28-35.

55. H. Rashmanlou, S. Samanta, M. Pal and R.A.Borzooei, A study on bipolar fuzzy graphs, Journal of Intelligent and Fuzzy Systems, DOI: 10.3233/IFS-141333.

56. A. Rosenfeld, Fuzzy Graphs, In Fuzzy Sets and their Applications to Cognitive and Decision Processes, Zadeh. L.A., Fu, K.S., Shimura, M., Eds ; Academic Press, New York (1975) 77-95.

57. S. Samanta and M. Pal, Fuzzy tolerance graphs, International Journal of Latest Trends in Mathematics, 1(2) (2011) 57-67.

58. S. Samanta and M. Pal, Bipolar fuzzy hypergraphs, International Journal of Fuzzy Logic Systems, 2(1) (2012) 17-28.

59. S. Samanta and M. Pal, Fuzzy k-competition graphs and p-competition fuzzy graphs, Fuzzy Inf. Eng., 5(2) (2013) 191-204.

60. S. Samanta and M. Pal, Some more results on bipolar fuzzy sets and bipolar fuzzy intersection graphs, The Journal of Fuzzy Mathematics, 22(2) (2014) 253-262.

61. S. Samanta and M. Pal, Irregular bipolar fuzzy graphs, International Journal of Applications of Fuzzy Sets, 2 (2012) 91-102.

62. E. Sampathkumar, $(1, k)$-Domination in a Graph, Jour. Math. Phy. Sci. 22 (5) (1988) 613-619.

63. A. Somasundaram and S. Somasundaram, Domination in fuzzy graphs-I, Pattern Recognition Letters 19 (1998) 787-791. 
64. A. Somasundaram, Domination in fuzzy graphs-II, J. Fuzzy Math. 13 (2) (2005) 281-288.

65. S. Sunitha and A. Vijaya Kumar, Some metric aspects of fuzzy graphs, In Proceedings of the Conference on Graph Connections, Allied Publishers (1999) 111-114.

66. P.J. Slater, Dominating and Reference Sets in graph, J.Math and Phy.Sci. 22 (1988) 445-455.

67. M.S. Sunitha and A. Vijaya Kumar, Information Sciences, 113 (1999) 293-300.

68. M.S. Sunitha and A. Vijayakumar, Complement of a fuzzy graph, Indian Journal of Pure and Applied Mathematics 33(9) (2002) 1451-1464.

69. M. Tom and M.S. Sunitha, Sum distance in fuzzy graphs, Annals of Pure and Applied Mathematics 7(2) (2014) 73-89.

70. Z. Tong, D. Zheng, An algorithm for finding the connectedness matrix of a fuzzy graph, Congr. Numer. 120 (1996) 189-192.

71. V.G. Vizing, The Cartesian Product of Graphs Vyc. Sis. 9 (1963) 30-43.

72. J. Xu, The use of fuzzy graphs in chemical structure research, in: D.H. Rouvry (Ed.), Fuzzy Logic in Chemistry, Academic Press, (1997) 249-282.

73. R.T. Yeh, S.Y. Bang, Fuzzy relations, Fuzzy relations, fuzzy graphs and their applications to clustering analysis, in: L.A. Zadeh, K.S. Fu, M. Shimura (Eds.), Fuzzy Sets and Their Applications, Academic Press, (1975)125-149.

74. L.A. Zadeh, Fuzzy sets, Information and Control 8 (1965) 338-353.

75. L.A. Zadeh, Towardageneralizedtheoryofuncertainty $(G T U)$ anoutline, Information Sciences 172 (12) (2005) 1-40.

76. L.A. Zadeh, Is there a need for fuzzy logic?, Information Sciences 178 (13) (2008) 2751-2779.

77. L.A. Zadeh, Fuzzy sets, Inform. Control, 8 (1965) 338-353. 